\title{
Stochastic Finite Element Method for analyzing static and dynamic pull-in of microsystems
}

\author{
Stephan D. A. Hannot ${ }^{1,3}$, Clemens V. Verhoosel $^{2}$ and Daniel \\ J. Rixen ${ }^{1}$ \\ ${ }^{1}$ Delft University of Technology, Department 3ME, Mekelweg 2, 2628 CD Delft, The \\ Netherlands \\ ${ }^{2}$ Eindhoven University of Technology, Department of Mechanical Engineering, PO Box 513, \\ 5600 MB Eindhoven, The Netherlands
}

E-mail: s.d.a.hannot@tudelft.nl

\begin{abstract}
Electro-mechanical sensors and actuators are a specific type of microsystems. The electrostatic pull-in value is one of the defining characteristics for these devices. Because the material and geometrical properties of micro fabricated systems are often very uncertain, this pull-in value can be subject to considerable variations. Therefore it is important to be able to estimate how uncertainty of mechanical properties propagates to the uncertainty of pull-in values. In this work the required design sensitivities of static and dynamic pull-in are derived. These sensitivities are used to perform a perturbation-based stochastic FEM analysis of an electromechanical device. This stochastic analysis consists of an uncertainty analysis and a reliability analysis. This stochastic analysis is validated by an expensive crude Monte Carlo computation.
\end{abstract}

\section{Introduction}

In the second half of the 20th century the invention of the integrated circuit sparked a rapid development of the new fabrication technologies, required to make those circuits smaller. At this moment the characteristic feature size of those integrated circuits is well below a micrometer. Those micro-fabrication techniques can be used to create miniaturized mechanical sensors and actuators as well, which led to the development of micro-electro-mechanical systems (MEMS)[1].

A subset of these MEMS, the electro-mechanical devices, use electrical potentials to deform a flexible part as is schematically shown in figure 1. An electric potential induces a charge difference between this deformable part and the grounded non deformable part. Due to the Coulomb forces the flexible part is attracted to the fixed part[2].

Charges within a conducting part tend to move to that point that is closest to a differently charged conductor. For instance the charges in the deformable conductor of figure 1 tend to move to the right bottom corner of that conductor. This effect is bigger when the distance between the conductors is smaller; hence the electric forces do not only depend on the applied potential difference, but also on the displacement of the deformable electrode. Both effects are non-linear.

3 Financial support of the MicroNed program is greatly acknowledged 

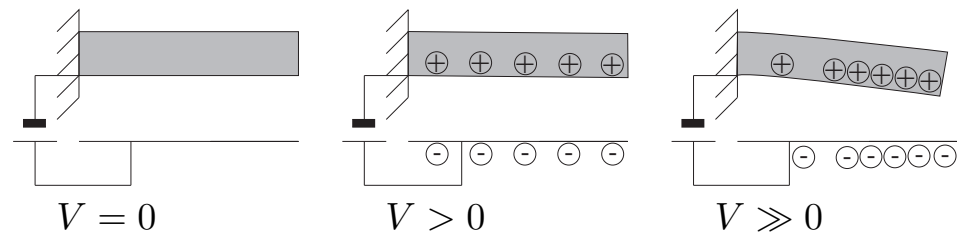

Figure 1. Illustration of electromechanical coupling

If the displacement of such a device is plotted versus the electric load there is maximum voltage for which there exists an equilibrium displacement. If the potential is increased beyond this point, there does not exist an equilibrium position anymore and the movable electrode will be pulled against the fixed one. This point is called the pull-in voltage[2]. The corresponding displacement is called the pull-in displacement and is in general smaller than the gap size.

This sudden instability, and the big difference between the stable equilibrium displacement before pull-in and the closed situation after pull-in, makes the pull-in voltage a sharp characteristic of an electro-mechanical microsystem. If no precautions are taken, a current starts flowing between the deformable and fixed electrode, which can potentially overheat the device. However, pull-in can be used for switching purposes as well. In both cases the pull-in voltage is a very critical parameter for the operation of the MEMS[3, 4].

When the voltage is applied with a sudden step, dynamic effects such as overshoot can cause the movable electrode to snap against the fixed one for a lower voltage than the static pull-in voltage, leading to the equally critical definition of the dynamic pull-in voltage [5].

Not surprisingly these pull-in voltages depend highly and nonlinearly on the geometry and material properties of the device. However, the material properties and dimensions of micro fabricated designs can be very unpredictable. This is more relevant for MEMS than for integrated circuits because MEMS designs use a wider and less standardized range of micro fabrication techniques $[6,7]$. Therefore it is important to determine how these uncertainties propagate through the design to the pull-in uncertainty.

The uncertainty is likely to be in the geometry of the device or in the constitutive equations. Crude Monte Carlo sampling can be used to determine the pull-in distribution. Monte Carlo sampling uses the principle that if enough models are generated using the proper distribution of the random parameters, the statistical moments of the outcome will converge. This is very expensive, but accurate, hence this approach will be used as a validation method in this work[8]. Perturbation-based approaches use sensitivities to compute the propagation of uncertainty and are computationally much cheaper $[9,10]$. The combination of the Finite Element Method with randomness as used in this paper is called stochastic FEM[11].

This paper starts with the FEM implementation of an electro-mechanical actuator, including the introduction of a test case. In section 3 the stochastic FEM is shortly recapitulated and in section 4 the required sensitivity computations are introduces. The stochastic FEM is applied on an electro-mechanical test case for validation in section 4 .

\section{FEM modeling of static and dynamic pull-in}

2.1. Coupled electromechanical equations

An electro-mechanically actuated microsystem can be partitioned into two domains: the conducting solid parts and the dielectric air region in between and around the solid parts. It is assumed that charges within the solid conductor can move fast enough to consider the solid to be an ideal conductor for the frequencies at which the systems is actuated. Therefore the electric potential within these conductors is constant and equal to their externally applied potential, limiting the electric domain to the air gap. Also it is assumed that the air is an ideal 
dielectric insulator, hence no charges are present or move inside the air gap.

Within this dielectric domain the electric potential can be described by Laplace's equation, which after Finite Element discretization yields a linear relation between the applied charge due to the boundary potentials and the potential within the electric domain[12]:

$$
\boldsymbol{q}=\boldsymbol{q}^{e x t}(\boldsymbol{u}, V)-\boldsymbol{K}^{v}(\boldsymbol{u}) \boldsymbol{v}=\mathbf{0}
$$

where $\boldsymbol{v}$ are the nodal electric potentials and $\boldsymbol{q}$ are nodal charges, induced by the boundary potential $V$. The matrix $\boldsymbol{K}^{v}$ describes the linear relation between charge and potential. The equations depend non linearly on the displacement of the solid parts $\boldsymbol{u}$ because those displacements change the shape of the electric domain. This defines the coupling from mechanical displacements to the electric potentials.

For the mechanical equations, describing the deformation, it is assumed that all electric forces are located at the boundary of the solid. Furthermore it assumed that mechanical forces due to air displacement or compression can be neglected. Is assumed that there exists a linear relation between the external forces and mechanical displacement:

$$
\boldsymbol{f}=\boldsymbol{f}^{e x t}(\boldsymbol{u}, \boldsymbol{v})-\boldsymbol{K}^{m} \boldsymbol{u}=\mathbf{0}
$$

where $\boldsymbol{K}^{m}$ is the mechanical stiffness matrix and $\boldsymbol{f}^{e x t}$ is the external force, in this case the electrostatic force.

This electric force follows from the electric field and is proportional to the square of the electric field $\boldsymbol{E}$ :

$$
\left|\boldsymbol{f}^{e x t}\right| \propto|\boldsymbol{E}|^{2},
$$

where the electric field is the gradient of the electric potential. After discretization, the electric force depends linearly on the electric field and non-linearly on the displacement.

To solve the coupled mechanical problem with an iterative procedure the tangent stiffness $\boldsymbol{K}$ has to be known [12]. Using this tangent stiffness, an increment in the solutions is obtained using:

$$
\boldsymbol{K} \Delta \boldsymbol{U}=\Delta \boldsymbol{F} .
$$

where:

$$
\boldsymbol{K}=\left[\begin{array}{cc}
\boldsymbol{K}^{m}-\frac{\partial \boldsymbol{f}^{e x t}}{\partial \boldsymbol{u}} & -\frac{\partial \boldsymbol{f}}{\partial \boldsymbol{v}} \\
-\frac{\partial \boldsymbol{q}}{\partial \boldsymbol{u}} & \boldsymbol{K}^{v}
\end{array}\right], \quad \Delta \boldsymbol{U}=\left[\begin{array}{c}
\Delta \boldsymbol{u} \\
\Delta \boldsymbol{v}
\end{array}\right], \quad \Delta \boldsymbol{F}=\left[\begin{array}{c}
\Delta \boldsymbol{f} \\
\Delta \boldsymbol{q}
\end{array}\right]
$$

\subsection{Static pull-in}

Pull-in is defined as the maximum voltage for which there are equilibrium solutions. Because a solution is described by the static state $\boldsymbol{U}$ this is the maximum of $V(\boldsymbol{U})$. Therefore at pull-in the derivative of $V$ with $\boldsymbol{U}$ must be zero. Because elements of $\boldsymbol{F}$ depend either linearly or quadratically on the applied voltage $V$, this means that:

$$
\frac{\partial \boldsymbol{F}}{\partial \boldsymbol{U}}=\boldsymbol{K}
$$

must be a singular matrix at pull-in. Hence the eigenvalue problem:

$$
\boldsymbol{K} \boldsymbol{\mu}=\lambda \boldsymbol{\mu}
$$

will return a zero lowest eigenvalue when $\boldsymbol{K}$ is evaluated at pull-in, and in that case the eigen mode $\boldsymbol{\mu}$ is known as the pull-in mode. This pull-in definition is very similar to the definition of limit point buckling [13]. 


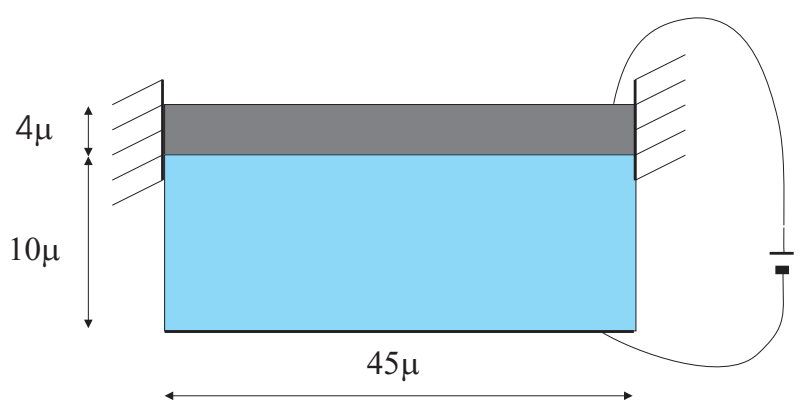

Figure 2. Reference problem setup.

\subsection{Dynamic pull-in}

In the dynamic case the response can become unbounded at voltages lower than the pull-in voltage, calling for the definition of a dynamic pull-in. A common way of defining dynamic pull-in is: The lowest voltage $V_{D P I}$ for which, when it is applied with a step from zero to $V_{D P I}$, the displacement response is unbounded $[4,5]$.

The way to detect if the current voltage estimate is below or above dynamic pull-in is by time integrating the equations of motion for about two oscillations. Without damping the transition is pretty sharp, thus when the applied voltage is above dynamic pull-in the displacement will become bigger than the gap within two oscillations. A bisection algorithm is used to pinpoint the dynamic pull-in voltage, which can be computationally expensive.

\subsection{Reference problem}

The test case will be a micro-bridge as shown in figure 2. It is meshed with 4 by 12 elements (12 beam elements 4 by 12 electric elements). The Young's modulus is chosen to be $E=1 \cdot 10^{5} \mathrm{~Pa}$ and the electric permittivity equal to the vacuum permittivity $\varepsilon=8.8541 \cdot 10^{-12} \mathrm{~F} / \mathrm{m}$.

The deterministic pull-in voltage is $V_{P I}=44.05$ volts. The static load displacement curve for this reference beam is shown in figure 3 . It shows the equilibrium solutions for different applied voltages. The maximum is the pull-in voltage.

The dynamic pull-in is $V_{D P I}=40.62$ volts. Figure 4 gives some step responses, above pullin the result diverges strongly. The maximum displacement that is reached during the stable transients is much bigger than the static pull-in displacement of about $3.5 \mu \mathrm{m}$. The dynamic pull-in is approximately $7 \%$ lower than the static pull-in.

\section{Stochastic FEM}

\subsection{Random variables}

It is assumed that some mechanical properties of the MEMS are randomly distributed parameters. Those properties are for instance the Young's modulus or the thickness of the device. Such a random property $\tilde{p}$ is indicated by a tilde. The most important characteristics of this random number are its mean value $\mu_{p}$ and its standard deviation from the mean $\sigma_{p}$, which describe the randomness. A non-dimensional estimation of the randomness is the coefficient of variation:

$$
V_{p}=\frac{\sigma_{p}}{\mu_{p}} .
$$

These definitions hold when there is only one random variable, which is constant throughout the design. When the device is rather big compared to characteristic length this might not hold. If that is the case the parameter is assumed to be a random field and the parameter of interest is a function of location: $\tilde{p}=\tilde{p}(x)$. 


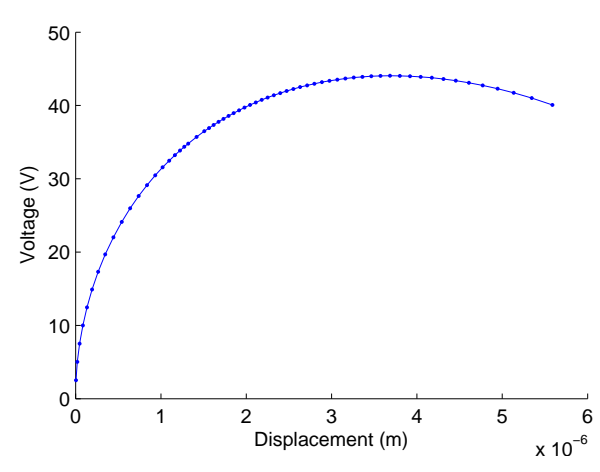

Figure 3. Applied static load versus the middle node displacement.

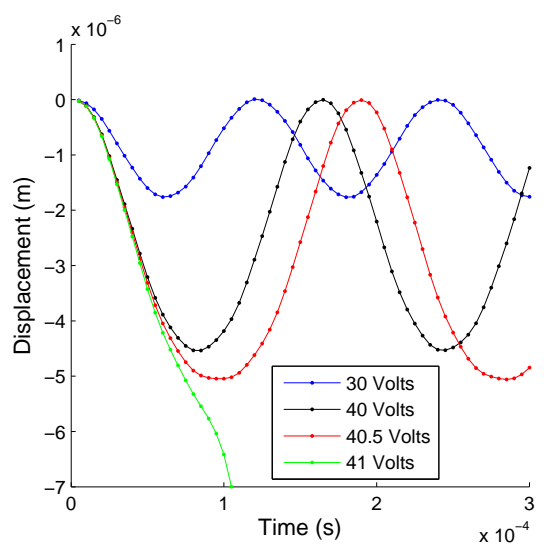

Figure 4. Step responses for different voltages.

Of course in a FEM setting this field has to be discretized. Here the used approach is to assume that within an element the parameter is uniform and equal to the value at the center of the element. This is the so called Midpoint method. This means that that we have $n$ different random parameters $\tilde{p}_{j}$, or a vector $\tilde{\boldsymbol{p}}$. However all with the same $\mu_{p_{j}}=\mu_{p}$ and $\sigma_{p_{j}}=\sigma_{p}$. However we also need to assume the dependency on each other. This is in line with experimental observations. But also because when it is assumed that all $\tilde{p}_{j}$ are totally unrelated, the problem becomes mesh dependent. Therefore it is assumed that:

$$
\Sigma_{p_{i} p_{j}}=\sigma_{p}^{2} e^{\left(-\frac{\left(x_{i}-x_{j}\right)^{2}}{l_{c}^{2}}\right)},
$$

where $l_{c}$ is a correlation length and $\Sigma_{p_{i} p_{j}}$ the covariance matrix.

This correlation length is the characteristic length that determines whether a random field approach or a uniform random number approach should be used. Basically a random field discretization is required when the device on the wafer is big compared to the wafer size. In that case properties tend to vary within the device.

\subsection{Uncertainty analysis}

To estimate the propagation of the uncertainty of a parameter $\tilde{\boldsymbol{p}}$, the perturbation technique is used[11,9]. Based on a Taylor expansion the mean value of the pull-in as a function of the random properties of $\tilde{p}$ are:

$$
\mu_{V_{P I}}=V_{P I}\left(\boldsymbol{\mu}_{p}\right)+\frac{1}{2} \Sigma_{p_{i} p_{j}} \frac{\partial^{2} V_{P I}}{\partial p_{i} \partial p_{j}},
$$

where it is seen that the mean value of the pull-in does not have to be equal to the deterministic pull-in value evaluated at the mean of the parameter $\tilde{p}$. The equation above is the second order estimate of the mean. If this correction based on the second order sensitivity is not computed, one finds the first order estimate.

Similarly:

$$
\Sigma_{V_{P I} V_{P I}}=\frac{\partial V_{P I}}{\partial p_{i}} \Sigma_{p_{i} p_{j}} \frac{\partial V P I}{\partial p_{j}} .
$$

which is the first order estimate of the variance of $\tilde{V}_{P I}$. 


\subsection{Reliability analysis}

Often design fails if $\tilde{V}_{P I}$ is bigger or smaller than a critical value $V_{c}$. In that case $\tilde{V}_{P I}$ should not exceed $V_{c}$. This defines the limit state function:

$$
\tilde{g}=V_{c}-\tilde{V}_{P I},
$$

and the chance on failure is defined as:

$$
\operatorname{Pr}[\tilde{g}>0] .
$$

There are many realizations of $\tilde{\boldsymbol{p}}$ that result in zero limit state. Therefore the failure chance is difficult to compute. However most of those realizations are very unlikely to happen, therefore within a first order reliability estimate it is assumed that the most likely $\boldsymbol{p}$ such that $g(\boldsymbol{p})=0$ provides sufficient information to estimate the overall failure chance. When assumed that $\tilde{\boldsymbol{p}}$ is normally distributed one can compute the probability that $\boldsymbol{p}$ is bigger than the one such that $g(\boldsymbol{p})=0[11]$.

\section{Sensitivity analysis}

Both the uncertainty and reliability analysis, presented above, require the computation of the sensitivities of the pull-in voltage. Here the sensitivities are computed for the static and dynamic case.

\subsection{Static pull-in}

Because static pull-in is very similar to limit point buckling, analogous to the buckling sensitivity the first order pull-in sensitivity can be computed with [14]:

$$
\frac{d V_{P I}}{d p}=-\frac{\boldsymbol{\mu}^{T} \frac{\partial \boldsymbol{F}}{\partial p}}{\boldsymbol{\mu}^{T} \frac{\partial \boldsymbol{F}}{\partial V}},
$$

evaluated at $\boldsymbol{U}_{P I}$ with $\boldsymbol{\mu}$ the pull-in mode. The derivation of this derivative used the fact that the lowest eigenvalue is zero. The derivative of $\boldsymbol{F}$ with the applied voltage $V$ is discussed in [15] and depends on $\boldsymbol{U}_{P I}$. The derivative of $\boldsymbol{F}$ with $p$ can be very simple if $p$ is parameter like the Young's modulus or beam element thickness. In that case only the mechanical part of $\boldsymbol{F}$ depends directly on $p$ :

$$
\frac{\partial \boldsymbol{F}}{\partial p}=\left[\begin{array}{c}
-\frac{\partial \boldsymbol{K}^{m}}{\partial p} \boldsymbol{u}_{P I} \\
\mathbf{0}
\end{array}\right]
$$

The second order sensitivity is computed by simply deriving (14):

$$
\frac{d^{2} V_{P I}}{d p^{2}}=\frac{d}{d p}\left(-\frac{\boldsymbol{\mu}^{T} \frac{\partial \boldsymbol{F}}{\partial p}}{\boldsymbol{\mu}^{T} \frac{\partial \boldsymbol{F}}{\partial V}}\right) .
$$

Using the product and chain rules one finds that the partial derivative of $\boldsymbol{\mu}$ with $p$ is required, for which Nelson's method can be used [16]. Also higher order partial derivatives of $\boldsymbol{F}$ with $p$ are needed, which can be derived analytically by looking at the implementation carefully. The last term needed is the derivative of $\boldsymbol{U}_{P I}$, while remaining on pull-in. This term is approximated using finite differences:

$$
\frac{d \boldsymbol{U}_{P I}}{d p}=\left.\frac{\Delta \boldsymbol{U}}{\Delta p}\right|_{V=\text { constant }}+\left.\frac{\Delta \boldsymbol{U}}{\Delta V}\right|_{p=\text { constant }} \frac{d V_{P I}}{d p},
$$

which is very efficient. Therefore the total computation of the second order sensitivity of pullin is a semi-analytical one. Those terms approximated above by finite differences can also be computed analytically[17]. 


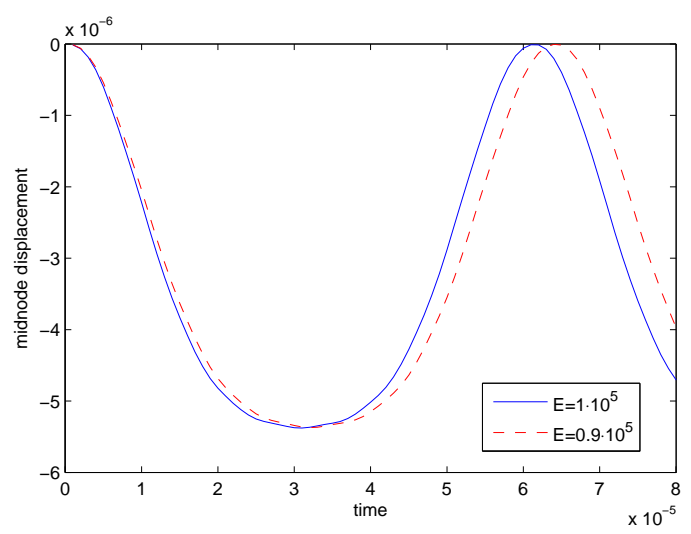

Figure 5. Transient results at the dynamic pull-in voltage

\subsection{Dynamic pull-in}

The stated definition of dynamic pull-in is less precise than that of static pull-in. For static pull-in it was known that the tangent stiffness matrix is singular, which enabled the sensitivity definition analogous to the limit point buckling one. However the analytical dynamic pull-in displacement for a 1D simplified model is independent of mechanical properties such as stiffness [18]. Is that also true for general FEM models?

With some heuristic reasoning it is possible to show that this claim is very likely to hold. When the dynamical pull-in is computed for the test case model with a Young's modulus of $E=0.9 \cdot 10^{5}$ instead of $E=1 \cdot 10^{5}$, dynamic pull-in decreases to 38.34 . When the transient response is computed for both moduli just below pull-in, the result of the middle node displacement are the curves in figure 5. The maximum displacement of both curves (different potential, different stiffness) is equal. This will not be the case for parameters that change the shape of the electric domain, effectively limiting the use of the method for dynamic pull-in to parameters as the elasticity modulus or the thickness of beam and plate elements.

When that statement is accepted, the point at which dynamic pull-in occurs can be defined as the voltage for which:

$$
f=u\left(V, p, t_{\max }\right)-u_{D P I}=0,
$$

where $t_{\max }$ is the time when the norm of the displacement is maximal during the transient, $u$ is the displacement of the middle node and $u_{D P I}$ the stiffness invariant pull-in displacement. The displacement $u$ is a function of the applied voltage step $V$, which at pull-in is assumed to be a function of the mechanical parameter $p$ of interest. The displacement is also directly a function of $p$.

This defines dynamic pull-in, but the end the purpose of this derivation is in how the dynamic pull-in changes with a mechanical parameter $p$. Therefore the derivative of $f$ is taken:

$$
\frac{d f}{d p}=\frac{\partial f}{\partial V} \frac{d V}{d p}+\frac{\partial f}{\partial p}+\frac{\partial f}{\partial t} \frac{d t_{\max }}{d p} .
$$

Because the variation of $V$ while remaining at pull-in is of interest, hence while $f$ remains zero, this derivative is restricted to zero values:

$$
\frac{d f}{d p}=\frac{\partial f}{\partial V} \frac{d V}{d p}+\frac{\partial f}{\partial p}+\left.\frac{\partial f}{\partial t} \frac{d t_{\max }}{d p}\right|_{D P I}=0,
$$

Furthermore at $f=0$ it reaches a maximum in time, hence the velocity of $f$ is zero:

$$
\frac{\partial f}{\partial t}=0
$$


thus the value computed by:

$$
\frac{d V_{D P I}}{d p}=-\left.\left(\frac{\partial f}{\partial V}\right)^{-1} \frac{\partial f}{\partial p}\right|_{f=0},
$$

will give the proper sensitivity. The two derivatives of $f$ are basically two derivatives of $f$ with a different parameter $p$. Using the chain rule this derivative simplifies to:

$$
\frac{\partial f}{\partial p}=\frac{\partial f}{\partial u} \frac{\partial u}{\partial p}=\frac{\partial u}{\partial p}=\frac{\partial u(t)}{\partial p} .
$$

Since $u$ is in essence an entry of $\boldsymbol{U}$ the goal is computing $\frac{\partial \boldsymbol{u}(t)}{\partial p}$. An efficient method is derived in [19]. There it is argued that at each instant in time:

$$
\frac{d^{2}}{d t^{2}}\left(\frac{\partial \boldsymbol{U}}{\partial p}\right)=\boldsymbol{M}^{-1}\left(\frac{\partial \boldsymbol{F}}{\partial p}-\boldsymbol{K}_{t} \frac{\partial \boldsymbol{U}}{\partial p}\right)
$$

where all the terms on the right-hand side depend on $\boldsymbol{U}$. During each time step of the time integration of the electro-mechanical equations one can use the knowledge of $\boldsymbol{U}$, to integrate the sensitivity as well. In that case one has all the prerequisites to compute the dynamic pull-in sensitivity. A second order sensitivity is cumbersome to compute, hence a second order estimate of the dynamic pull-in mean value is impractical.

\section{Results}

\subsection{Stochastic analysis of static pull-in}

The random properties of $\tilde{E}$ are: $\mu_{E}=1 \cdot 10^{5} \mathrm{~Pa}, \sigma_{E}=1 \cdot 10^{4} \mathrm{~Pa}$ and $V_{E}=0.1$. The correlation length is one third of the beam length: $15 \cdot 10^{-6} \mathrm{~m}$.

This gives with the perturbation approach

$$
\mu_{V_{P I}}=V_{P I}\left(\boldsymbol{\mu}_{h}\right)+\frac{1}{2} \Sigma_{E_{i} E_{j}} \frac{\partial^{2} V_{P I}}{\partial E_{i} \partial E_{j}}=44.05-0.056=44.00 \mathrm{~V} .
$$

The stochastic mean voltage is lower than the deterministic pull-in voltage due to the negative second order sensitivity. This property should be considered when designing MEMS. The standard deviation is:

$$
\sigma_{V_{P I}}=\sqrt{\frac{\partial V_{P I}}{\partial h_{i}} \Sigma_{h_{i} h_{j}} \frac{\partial V_{P I}}{\partial h_{j}}}=1.44 \mathrm{~V},
$$

hence the coefficient of variation is:

$$
V_{V_{P I}}=0.033 \text {. }
$$

Thus the pull-in voltage has a lower coefficient of variation than the elasticity modulus. Thus relative stiffness variations are damped by the device to give smaller relative pull-in variations.

A Monte Carlo simulation with 4638 random evaluations gave:

$$
\begin{array}{r}
\mu_{V_{P I}}=43.98 \mathrm{~V}, \\
\sigma_{V_{P I}}=1.44 \mathrm{~V}, \\
V_{V_{P I}}=0.033 .
\end{array}
$$

These numbers are in excellent agreement. From figure 6 it can be seen that the mean has converged to 44.0. Furthermore it is clearly below 44.05 as predicted by the second order 


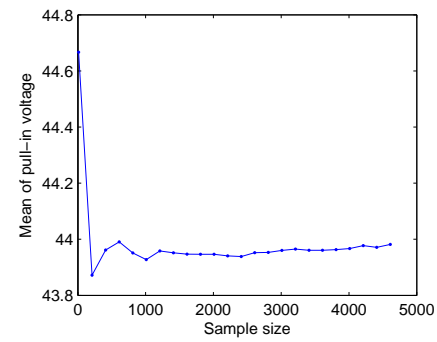

Figure 6. Convergence of the mean with sample size.

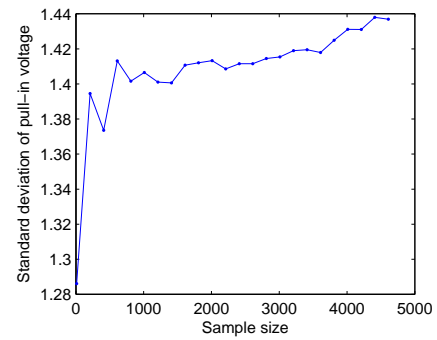

Figure 7. Convergence of the standard deviation with sample size.

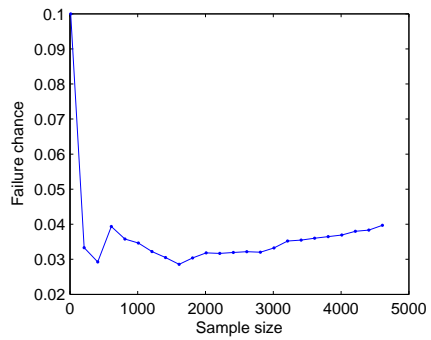

Figure 8. Convergence of the failure chance with sample size.

contribution in the perturbation approach. The standard deviation in figure 7 is less well converged, but seems to be within the band of 1.4 to 1.5. The sensitivity computation costs about the same time as one pull-in evaluation, hence the perturbation-based stochastic FEM method is computationally much cheaper.

For this reliability analysis it is assumed that this electro-mechanically actuated device is a switch, which fails if the switching voltage is too high (above 46.5). When the failure chance is determined with the sensitivity based approach, this yields:

$$
\operatorname{Pr}\left[V_{P I}>46.5\right]_{\text {per }}=0.0397,
$$

which required only 5 function evaluations to obtain with the HL-RF algorithm[11]. The Monte Carlo simulation of 4638 realizations, which converged as shown in figure 8, gave:

$$
\operatorname{Pr}\left[V_{P I}>46.5\right]_{m c}=0.040 .
$$

Also these results are in very good agreement.

\subsection{Stochastic analysis of dynamic pull-in}

Because an accurate dynamic pull-in computation is very expensive and a higher number of stochastic variables requires more accuracy than a low number of stochastic variables it is assumed in this section that $\tilde{E}$ is random, but constant along the beam for each realization. The elasticity modulus is assumed to have a mean value of $\mu_{E}=1 \cdot 10^{5} \mathrm{~Pa}$ and a standard deviation of $\sigma_{E}=1 \cdot 10^{4} \mathrm{~Pa}$.

The perturbation-based uncertainty analysis gives:

$$
\begin{gathered}
\mu_{V_{D P I}}=40.42 \mathrm{~V}, \\
\sigma_{V_{D P I}}=2.02 \mathrm{~V},
\end{gathered}
$$

respectively for the first order mean value of dynamic pull-in and perturbation-based standard deviation is.

Also this dynamic analyses requires a Monte Carlo validation. Hence a sample of 13352 realizations is used for validation. This gives:

$$
\mu_{V_{D P I}}=40.38 \mathrm{~V},
$$

which is a little bit lower that the deterministic value above, showing the usefulness of second order sensitivities. The Monte Carlo standard deviation is:

$$
\sigma_{V_{D P I}}=2.03 \mathrm{~V},
$$


which is close to the perturbation SFEM approach.

For the static problem the failure criterion was: $V_{P I}>46.5$. However this was based on the assumption that it would fail if the switching voltage is too high. Often electro-mechanical devices are not switches but sensors which are actuated to tune the resonance frequency. In that case pull-in is something to be avoided and the device fails if pull-in is too low. For instance if $V_{D P I}<37$, which gives the following failure chances for the perturbation and Monte Carlo approaches respectively:

$$
\begin{aligned}
& \operatorname{Pr}\left[V_{D P I}<37\right]_{p e r}=0.0524 . \\
& \operatorname{Pr}\left[V_{D P I}<37\right]_{m c}=0.0515 .
\end{aligned}
$$

The numbers are again close, therefore validating the perturbation approach.

The elasticity modulus is assumed to be normally distributed for computation of this failure chance. The perturbation-based failure chance is obtained by computing the elasticity value for which the pull-in voltage is equal to the critical voltage $\left(E=8.38 \cdot 10^{5} \mathrm{~Pa}\right)$. After which the failure chance is computed as the chance that the normally distributed elasticity is smaller than the critical one. This is not the same as assuming a normally distributed pull-in voltage around the 40.42 mean value, which would give a too low failure chance of 0.0452 .

\section{Conclusions}

Analytical first order sensitivities for both static and dynamic pull-in were derived as well as the second order sensitivity of the static pull-in value. These sensitivities can be used for perturbation-based stochastic finite element methods. In this work a pull-in uncertainty analysis and a pull-in based reliability analysis of a mirco-bridge were performed. These perturbation-based results were validated by crude Monte Carlo simulations, hence perturbationbased methods can be used to estimate the stochastic behavior of electro-mechanically actuated devices. The computational advantage is huge, since they are much cheaper than the Monte Carlo methods.

\section{References}

[1] Pasquale M 2003 Sensors \& Actuators: A. Physical 106 142-148

[2] Nathanson H, Newell W, Wickstrom R and Davis Jr J 1967 Electron Devices, IEEE Transactions on 14 $117-133$

[3] Rebeiz G 2003 RF MEMS: theory, design, and technology (John Wiley and Sons)

[4] Elata D and Bamberger H 2006 Microelectromechanical Systems, Journal of 15 131-140

[5] Rochus V, Rixen D and Golinval J 2005 Nonlinear Analysis 63 1619-1633

[6] Cho S and Chasiotis I 2007 Experimental Mechanics 47 37-49

[7] Madou M 1997 Fundamentals of Microfabrication (New York: CRC Press) iSBN 0-8493-9451-1

[8] Li J and Chen J 2009 Stochastic Dynamics of Structures (Wiley-Blackwell)

[9] Schuëller G 2006 Archive of Applied Mechanics 75 755-773

[10] Rahman S and Rao B 2001 Int. Journal for Numerical Methods in Engineering 50 1961-1991

[11] Gutierrez M and Krenk S 2004 Stochastic Finite Element Methods Encyclopedia of Computational Mechanics, volume 2: Solids and Structures ed de Borst R, Stein E and Hughes T (Wiley) pp 657-681

[12] Rochus V, Rixen D and Golinval J 2006 Int. Journal for Numerical Methods in Engineering 65 461-493

[13] Hannot S and Rixen D 2009 Numerical Modeling of the Electromechanical Interaction in MEMS Advanced Computational Methods in Science and Engineering ed Koren B and Vuik K (Springer) pp 315-342

[14] de Boer H and van Keulen F 2000 Int. Journal for Numerical Methods in Engineering 37 6961-6980

[15] Lemaire E, Rochus V, Golinval J and Duysinx P 2008 Computer Methods in Applied Mechanics and Engineering 197 4040-4050

[16] Nelson R 1976 AIAA Journal 14 1201-1205

[17] Verhoosel C 2009 Multiscale and probabilistic modelling of micro electromechanical systems (Netherlands: Delft University of Technology, PhD Thesis)

[18] Rochus V 2006 Finite element modeling of strong electro-mechanical coupling in MEMS (Belgium: Universite de Liege, PhD Thesis)

[19] Brüls O and Eberhard P 2008 Int. Journal for Numerical Methods in Engineering 74 